\section{CONCLUSIONS}

We have combined the idea of a central incision to provide safer passage of the pectus bar with that of 3-point fixation to limit displacement of the pectus bar to create a modification of the Nuss procedure, with success in more than 80 cases.

\section{References}

1. Nuss D. Minimally invasive surgical repair of pectus excavatum. Semin Pediatr Surg. 2008;17:209-17
2. Ohno K, Nakamura T, Azuma T, Yamada H, Hayashi H, Masahata K. Modification of the Nuss procedure for pectus excavatum to prevent cardiac perforation. J Pediatr Surg. 2009;44:2426-30.

3. Johnson WR, Fedor D, Singhal S. A novel approach to eliminate cardiac perforation in the Nuss procedure. Ann Thorac Surg. 2013;95:1109-11.

4. Kim do H, Hwang JJ, Lee MK, Lee DY, Paik HC. Analysis of the Nuss procedure for pectus excavatum in different age groups. Ann Thorac Surg. 2005;80: 1073-7.

5. Tedde ML, Campos JR, Das-Neves-Pereira JC, Abrāo FC, Jatene FB. The search for stability: bar displacement in three series of pectus excavatum patients treated with the Nuss technique. Clinics (Sao Paulo). 2011;66:1743-6.

6. Hebra A, Gaudere MW, Tagge EP, Adamson WT, Othersen HB Jr. A simple technique for preventing bar displacement with the Nuss repair of pectus excavatum. J Pediatr Surg. 2001;36:1266-8

\title{
Reconstructive options for gastroesophageal junction adenocarcinoma after Roux-en-Y gastric bypass
}

\author{
Afif N. Kulaylat, MD, Sunny Sahajwani, MD, MHS, Kevin F. Staveley-O'Carroll, MD, PhD, and \\ Jussuf T. Kaifi, MD, PhD, Hershey, Pa
}

In Western countries, esophagogastric cancers have migrated toward the gastroesophageal junction (GEJ), with adenocarcinoma the predominating histologic type. GEJ cancers are the fastest growing cancers in the United States. ${ }^{1,2}$ Cancer development after Roux-en-Y gastric bypass (RNYGB) is not well characterized. Altered anatomy poses technical challenges for surgical resection. We report our reconstructive experience with GEJ adenocarcinoma diagnosed after RNYGB.

\section{CLINICAL SUMMARIES}

Between 2008 and 2012, a total of 271 consecutive patients underwent resection with curative intent for GEJ adenocarcinoma at our institution. Two patients $(0.7 \%)$ were encountered with GEJ cancers after RNYGB and reviewed with institutional review board approval.

\section{Patient 1}

A 62-year-old man (body mass index [BMI], $40 \mathrm{~kg} / \mathrm{m}^{2}$ ) with history of smoking and gastroesophageal reflux disease who had undergone RNYGB 6 years earlier

\footnotetext{
From the Program for Liver, Pancreas, and Foregut (Lung and Esophageal) Tumors, Section of Surgical Oncology, Department of Surgery, Penn State Milton S. Hershey Medical Center, Hershey, Pa.

Disclosures: Authors have nothing to disclose with regard to commercial support.

Received for publication March 5, 2013; revisions received April 15, 2013; accepted for publication July 9, 2013; available ahead of print Aug 28, 2013.

Address for reprints: Afif N. Kulaylat, MD, Section of Surgical Oncology, Department of Surgery, Penn State Milton S. Hershey Medical Center, Hershey, PA 17033 (E-mail: akulaylat@hmc.psu.edu).

J Thorac Cardiovasc Surg 2013;146:1296-8

0022-5223/\$36.00

Copyright (c) 2013 by The American Association for Thoracic Surgery

http://dx.doi.org/10.1016/j.jtcvs.2013.07.007
}

(initial BMI, $60 \mathrm{~kg} / \mathrm{m}^{2}$ ) was seen for epigastric pain and gastrointestinal bleeding. Evaluation by esophagogastroduodenoscopy and endoscopic ultrasonography with biopsy demonstrated poorly differentiated invasive adenocarcinoma of the distal esophagus (Siewert type I) with periesophageal and gastric lymph node involvement. Neoadjuvant chemoradiation achieved moderate response, as seen on positron emission tomography and computed tomography (CT). He subsequently underwent an open transhiatal complete esophagectomy with gastric pouchectomy. The proximal alimentary Roux limb was resected, with a gastric conduit fashioned from the remnant. Continuity was restored by jejunojejunostomy (abandoning the Roux-en$\mathrm{Y}$ ), and a cervical esophagogastrostomy was created (Figure 1). Pathology showed a completely resected T3N1 adenocarcinoma. He received adjuvant chemotherapy (subsequent BMI, $32 \mathrm{~kg} / \mathrm{m}^{2}$ ). Recurrent disease was discovered in his liver on surveillance positron emission tomography and CT after 15 months, and his overall survival after resection was 19 months.

\section{Patient 2}

A 54-year-old woman (BMI, $34 \mathrm{~kg} / \mathrm{m}^{2}$ ) with a history of smoking and gastroesophageal reflux disease who had undergone RNYGB 7 years earlier (initial BMI, $55 \mathrm{~kg}$ / $\mathrm{m}^{2}$ ) had dysphagia and weight loss. Esophagogastroduodenoscopy revealed a $2-\mathrm{cm}$ gastric pouch ulcer, with biopsy demonstrating a poorly differentiated adenocarcinoma (Siewert type III). Intraoperatively, the cancer invaded the remnant stomach from the gastric pouch. With en bloc resection of the remnant, a total gastrectomy was performed, leaving a duodenal stump. The previous Roux 
INITIAL ANATOMY

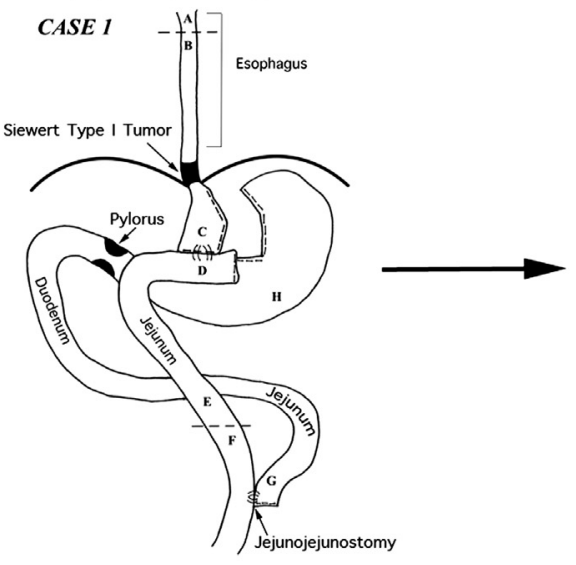

RESECTION/SPECIMEN

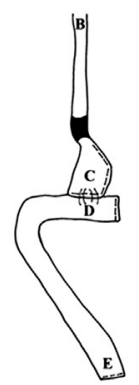

\section{RECONSTRUCTION}

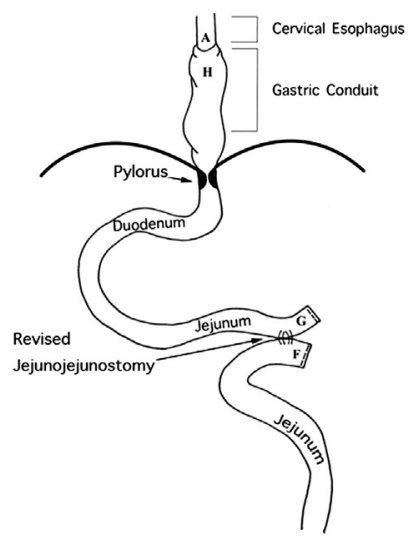

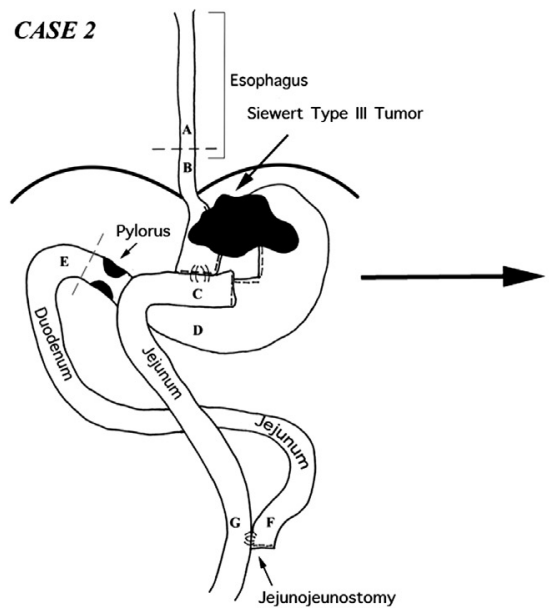
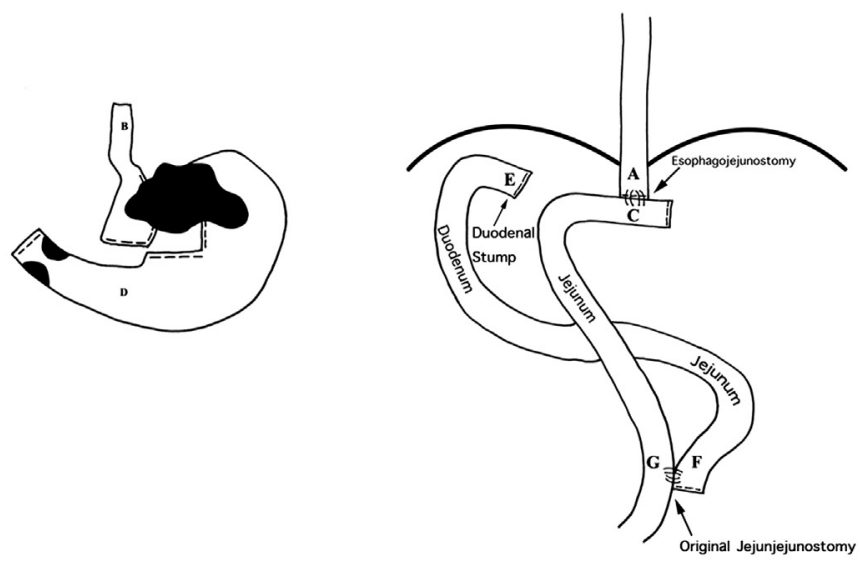

FIGURE 1. Original Roux-en-Y gastric bypass anatomy with location of gastroesophageal junction cancer for each case, followed by resected specimen and final reconstruction anatomy. Case 1: $A$, cervical esophagus; $B$, intrathoracic esophagus; $C$, gastric pouch; $D$, alimentary jejunal limb of the gastrojejunostomy, $E$, alimentary jejunal limb; $F$, alimentary jejunal limb of the jejunojejunostomy; $G$, biliopancreatic jejunal limb of the jejunojejunostomy; $H$, gastric remnant. Case 2: $A$, intrathoracic esophagus; $B$, distal esophagus; $C$, alimentary jejunal limb of the gastrojejunostomy; $D$, gastric remnant; $E$, Pars I duodeni; $F$, biliopancreatic jejunal limb of the jejunojejunostomy; $G$, alimentary jejunal limb of the jejunojejunostomy.

limb was resected, and a novel Roux-en-Y reconstruction was achieved with end-to-side esophagojejunostomy (Figure 1). Final pathology showed T4N1M0 signet cell adenocarcinoma. She received adjuvant chemoradiation (subsequent BMI, $20 \mathrm{~kg} / \mathrm{m}^{2}$ ). She has been recurrence free for 20 months.

\section{DISCUSSION}

There are fewer than 25 published reports of GEJ adenocarcinoma in patients with gastric bypass procedures, including RNYGB, loop gastric bypass, vertical band gastroplasty, and band procedures. ${ }^{1}$ Reports of transhiatal esophagectomy with cervical esophagogastrostomy with the remnant as a conduit are limited. ${ }^{2}$ Ivor Lewis esophagogastrectomy represents the predominant surgical approach. Patient 2 is unique in that invasion from the gastric pouch into the remnant rendered both sections unsuitable for reconstruction, necessitating a
Roux-en-Y esophagojejunostomy. Fortunately, it was a Siewert type III adenocarcinoma of the GEJ, so esophagectomy was not necessary to achieve adequate oncologic resection.

Technical considerations include preoperative localization with esophagogastroduodenoscopy and positron emission tomography and $\mathrm{CT}$ and intraoperative confirmation to dictate extent of surgical resection and reconstructive options. We recommend dissection of the remnant before the cancer resection to verify suitability as a conduit. The remnant can be tubularized in the standard fashion with staplers. In patient 1 , the majority of the remnant fundus was preserved, with comparable length to a normal gastric conduit for a side-to-side stapled cervical anastomosis. With transhiatal esophagectomy and remnant pull-up, we recommend jejunojejunostomy revision to restore functional small bowel length. 
If the remnant is unsuitable (such as in patient 2) or the length is insufficient, options include transthoracic (Ivor Lewis) anastomosis, esophagojejunostomy, or alternative conduits from jejunum or colon. ${ }^{3,4}$ The extent of esophageal resection (intraoperative frozen examination of esophageal margin is critical), anatomic considerations and surgeon preference and comfort should guide reconstruction. Concerning other key steps of the operation, particularly lymphadenectomy, standard surgical approaches for gastroesophageal cancers are followed.

Adenocarcinoma has also been described in the remnant, with abdominal pain the usual presenting symptom. ${ }^{5}$ CT or magnetic resonance imaging scans should be the primary screening modalities but may be inconclusive at early stages. Techniques including double balloon enteroscopy, laparoscopic transgastric endoscopy, and percutaneous gastrostomy tube placement offer access for evaluation.

By the nature of their comorbidities, primarily obesity and gastroesophageal reflux disease, the gastric bypass population are at higher risk for GEJ adenocarcinoma. ${ }^{2}$ Acid and biliary reflux into the esophagus are implicated in the development of metaplasia and subsequent cancer. ${ }^{2}$ RNYGB has previously been advocated as the ultimate antireflux procedure. ${ }^{2}$ The pathophysiology of GEJ adenocarcinoma in the altered anatomy of the RNYGB is, however, incompletely understood.

With the increasing incidence of GEJ adenocarcinomas and the rise in bariatric procedures, surgeons will encounter more patients for curative resection of esophagogastric cancers in the context of altered and complex bariatric gastrointestinal anatomy.

\section{References}

1. Kuruba R, Jawad M, Karl RC, Murr MM. Technique of resection of esophageal adenocarcinoma after Roux-en-Y gastric bypass and literature review of esophagogastric tumors after bariatric procedures. Surg Obes Relat Dis. 2009;5: 576-81.

2. Melstrom LG, Bentrem DJ, Salvino MJ, Blum MG, Talamonti MS, Printen KJ. Adenocarcinoma of the gastroesophageal junction after bariatric surgery. Am J Surg. 2008;196:135-8.

3. Ascioti AJ, Hofstetter WL, Miller MJ, Rice DC, Swisher SG, Vaporciyan AA, et al. Long-segment, supercharged, pedicled jejunal flap for total esophageal reconstruction. J Thorac Cardiovasc Surg. 2005;130:1391-8.

4. Avella D, Garcia L, Hartman B, Kimchi E, Staveley-O'Carroll K. Esophageal extension encountered during transhiatal resection of gastric or gastroesophageal tumors: attaining a negative margin. J Gastrointest Surg. 2009;13:368-73.

5. Watkins BJ, Blackmun S, Kuehner ME. Gastric adenocarcinoma after Roux-en-Y gastric bypass: access and evaluation of excluded stomach. Surg Obes Relat Dis. 2007;3:644-7. 